\title{
Temperature dependence of the dynamics of the first image-potential state on $\operatorname{Ag}(111)$
}

\author{
S. S. Tsirkin, ${ }^{1,2}$ S. V. Eremeev, ${ }^{1,3}$ E. V. Chulkov, ${ }^{2,4}$ M. Marks, ${ }^{5}$ K. Schubert, ${ }^{5}$ J. Güdde, ${ }^{5}$ and U. Höfer ${ }^{2,5}$ \\ ${ }^{1}$ Tomsk State University, Tomsk, 634050, Russia \\ ${ }^{2}$ Donostia International Physics Center (DIPC), 20018 San Sebastián/Donostia, Basque Country, Spain \\ ${ }^{3}$ Institute of Strength Physics and Materials Science, pr. Academicheskii 2/4, Tomsk, 634021, Russia \\ ${ }^{4}$ Departamento de Física de Materiales UPV/EHU, Centro de Física de Materiales CFM-MPC \\ and Centro Mixto CSIC-UPVIEHU, 20080 San Sebastián/Donostia, Basque Country, Spain \\ ${ }^{5}$ Fachbereich Physik und Zentrum für Materialwissenschaften, Philipps-Universität, D-35032 Marburg, Germany
}

(Received 14 June 2012; published 14 August 2012)

\begin{abstract}
The temperature dependence of the dynamics of electrons in the $n=1$ image potential state on the $\operatorname{Ag}(111)$ surface has been investigated by means of time-resolved two-photon photoemission spectroscopy and many-body calculations. We show that the decay rate of electrons in this state grows linearly with temperature. The thermal shortening of the lifetime is caused by the increase of the electron-electron scattering rate, due to deeper penetration of the image state wave function into the bulk metal at higher temperature. The electron-phonon scattering in this state is found to be small.
\end{abstract}

DOI: 10.1103/PhysRevB.86.085424

PACS number(s): 73.20.-r, 72.10.-d, 79.60.Bm, 78.47.J-

\section{INTRODUCTION}

Lifetimes of electronic excitations are crucial for many physical and chemical phenomena, such as charge and energy transport, ${ }^{1}$ interaction of atoms and molecules with the surface, ${ }^{2}$ and catalytic reactions. ${ }^{3}$ At metal surfaces, in addition to bulk electronic states, single-particle excitations occur in intrinsic surface states ${ }^{4,5}$ and in image-potential states. ${ }^{6-8}$ Surface states arise from the breaking of the crystal symmetry along the surface normal and are localized near the outermost atomic layer. Image-potential states are localized on the vacuum side of the surface. They are formed by the image potential, originating from the attractive interaction of the electron with the polarization charge. Far from the surface plane this potential converges asymptotically to the classical image potential, which is inversely proportional to the distance from the image plane. In the vicinity of the $\bar{\Gamma}$ point of the surface Brillouin zone image-potential states form a Rydberg-like series, converging to the vacuum level $E_{\mathrm{vac}}: 9,10$

$$
E_{n}\left(\mathbf{k}_{\|}\right)=E_{\mathrm{vac}}-\frac{0.85 \mathrm{eV}}{(n+a)^{2}}+\frac{\hbar^{2} \mathbf{k}_{\|}^{2}}{2 m_{n}^{*}},
$$

where $n=1,2, \ldots$ is the principal quantum number and $a$ is the quantum defect. The dispersion of image-potential states with the wave vector $\mathbf{k}_{\|}$in the surface plane is free-electronlike with effective masses $m_{n}^{*}$ close to the free-electron mass $m_{0}$. Due to their well-defined energy spectrum, which weakly depends on the microscopic details of the surface under consideration, image-potential states have been widely used as a model system for both theoretical and experimental investigations of the dynamics of electronic excitations at metal surfaces. ${ }^{11}$

The total decay rate (or linewidth) $\Gamma=\hbar / \tau$ ( $\tau$ being the lifetime) of an electronic excitation is determined by the contributions of four different processes: inelastic electron-electron scattering $\left(\Gamma_{e-e}\right)$, electron-phonon $(e-\mathrm{ph})$ interaction $\left(\Gamma_{e-\mathrm{ph}}\right)$, electron-defect scattering $\left(\Gamma_{e-d}\right)$, and energy-conserving resonant one-electron tunneling into the bulk $\left(\Gamma_{e-e}^{1 e}\right)$. The latter process contributes to the decay rate only when the initial electronic state is degenerate with projected bands of the bulk metal. ${ }^{12-14}$

The temperature dependence of the dynamics of electrons and holes in the surface states has been extensively studied both theoretically and experimentally on various metal surfaces, ${ }^{15-33}$ but only few works considered the temperature dependence of the image state lifetimes..$^{20,34-36}$

The thermal change of the decay rate is usually attributed to the $e$-ph interaction ${ }^{15,22,27,28,32,35-38}$ and scattering on thermally activated defects, ${ }^{19,24}$ while the electron-electron scattering contribution is usually assumed to be independent of temperature. This approximation is justified for the surface states, as far as $e$-ph interaction is rather strong in these states and $\Gamma_{e-\mathrm{ph}}$ often exceeds $\Gamma_{e-e}$ at room temperature. However, $e$-ph scattering in image-potential states has been predicted to be quite weak. ${ }^{34-36}$ The theoretical $e$-ph coupling parameter is typically $\lambda_{e-\text { ph }}<10^{-2}$, which cannot account for the thermal decrease of the $n=1$ image state lifetime from $22 \pm 3$ fs at $25 \mathrm{~K}$ to $14 \pm 3 \mathrm{fs}$ at $350 \mathrm{~K}$ observed in two-photon photoemission for a $\mathrm{Cu}(111)$ surface. ${ }^{20}$ Hence, in order to investigate theoretically the temperature dependence of the decay rate of electrons in image-potential states, it is necessary to take into account the temperature dependence of the inelastic electron-electron contribution.

In this article we present a comprehensive study of the temperature dependence of the dynamics of electrons in the $(n=1)$ image-potential state on $\operatorname{Ag}(111)$ by means of timeresolved two-photon photoemission spectroscopy (2PPE) and theoretical many-body calculations. Photoemission experiments reveal a temperature dependence of the energy and dynamics of the first image-potential state. The image-potential state shifts to lower energies with increasing temperature. In addition, a decrease of the inelastic lifetime is observed. We perform many-body calculations of the electron-electron and electron-phonon contributions to the decay rate of the $(n=1)$ state in the temperature range from 0 to $300 \mathrm{~K}$. We account for the temperature dependence of $\Gamma_{e-e}$ by taking into consideration the experimentally measured temperature dependence of the band structure. 
In Sec. II we outline the theoretical methods used to calculate the decay rate. Experimental techniques are described in Sec. III. The results of the experiments and calculations are presented and discussed in Sec. IV. The article is summarized in Sec. V. Unless otherwise explicitly stated, atomic units are used in the equations, i.e., $\hbar=m_{0}=e=1$.

\section{THEORY}

\section{A. Inelastic electron-electron scattering}

The calculations of the inelastic electron-electron scattering are performed in the self-energy formalism of many-body theory using the GW approximation. ${ }^{39}$ The method is described in detail elsewhere, ${ }^{11}$ and here we give just a brief overview. Within this formalism the inelastic electron-electron scattering contribution to the decay rate $\Gamma_{e-e}$ of the electronic state in band $i$ with wave function $\Psi_{i, \mathbf{k}_{i}}$, energy $E_{i, \mathbf{k}_{i}}$, and wave vector $\mathbf{k}_{i}$ is obtained as the projection of the imaginary part of the self-energy operator $\Sigma$ onto this state:

$$
\begin{aligned}
\Gamma_{\mathrm{e}-\mathrm{e}}= & -2\left\langle\Psi_{i, \mathbf{k}_{i}}|\operatorname{Im} \Sigma| \Psi_{i, \mathbf{k}_{i}}\right\rangle \\
= & -2 \sum_{f, \mathbf{k}_{f}}^{E_{F}<E_{f, \mathbf{k}_{f}}<E_{i, \mathbf{k}_{i}}} \iint\left[\Psi_{i, \mathbf{k}_{i}}^{*}(\mathbf{r}) \Psi_{f, \mathbf{k}_{f}}(\mathbf{r})\right. \\
& \left.\times \operatorname{Im} W\left(\mathbf{r}, \mathbf{r}^{\prime},\left|E_{i, \mathbf{k}_{i}}-E_{f, \mathbf{k}_{f}}\right|\right) \Psi_{f, \mathbf{k}_{f}}^{*}\left(\mathbf{r}^{\prime}\right) \Psi_{i, \mathbf{k}_{i}}\left(\mathbf{r}^{\prime}\right)\right] d \mathbf{r} d \mathbf{r}^{\prime}
\end{aligned}
$$

Here the self-energy is represented by the first term of the expansion in terms of the screened Coulomb interaction $W$, which is calculated within the random phase approximation. The summation is carried out over all final electronic states $\left(f, \mathbf{k}_{f}\right)$ with energies between the Fermi energy $E_{F}$ and the energy of the initial state $E_{i, \mathbf{k}_{i}}$. Thus, the many-body decay rate is determined by three main factors: (i) the phase space of the final states $\left(f, \mathbf{k}_{f}\right)$, (ii) the overlap between the wave functions of the initial and final states, and (iii) the magnitude of the imaginary part of the screened Coulomb interaction $\operatorname{Im} W$. The latter is given in linear response theory by

$$
\begin{aligned}
W\left(\mathbf{r}, \mathbf{r}^{\prime} ; \omega\right)= & V\left(\mathbf{r}-\mathbf{r}^{\prime}\right)+\iint\left[V\left(\mathbf{r}-\mathbf{r}_{1}\right)\right. \\
& \left.\times \chi\left(\mathbf{r}_{1}, \mathbf{r}_{2} ; \omega\right) V\left(\mathbf{r}_{2}-\mathbf{r}^{\prime}\right)\right] d \mathbf{r}_{1} d \mathbf{r}_{2},
\end{aligned}
$$

where $V\left(\mathbf{r}-\mathbf{r}^{\prime}\right)$ is the bare Coulomb interaction and $\chi\left(\mathbf{r}_{1}, \mathbf{r}_{2} ; \omega\right)$ is the density-density response function of the interacting electron system, which is evaluated from the equation

$$
\begin{aligned}
\chi\left(\mathbf{r}_{1}, \mathbf{r}_{2} ; \omega\right)= & \chi^{0}\left(\mathbf{r}_{1}, \mathbf{r}_{2} ; \omega\right)+\iint\left[\chi^{0}\left(\mathbf{r}_{1}, \mathbf{r}_{3} ; \omega\right)\right. \\
& \left.\times V\left(\mathbf{r}_{3}-\mathbf{r}_{4}\right) \chi\left(\mathbf{r}_{4}, \mathbf{r}_{2} ; \omega\right)\right] d \mathbf{r}_{3} d \mathbf{r}_{4}
\end{aligned}
$$

Here $\chi^{0}\left(\mathbf{r}_{1}, \mathbf{r}_{2} ; \omega\right)$ is the density-density response function of a noninteracting electron system:

$$
\begin{aligned}
\chi^{0}\left(\mathbf{r}_{1}, \mathbf{r}_{2} ; \omega\right)= & 2 \sum_{\alpha, \mathbf{k}_{\alpha}} \sum_{\beta, \mathbf{k}_{\beta}} \frac{\theta\left(E_{F}-E_{\alpha \mathbf{k}_{\alpha}}\right)-\theta\left(E_{F}-E_{\beta \mathbf{k}_{\beta}}\right)}{E_{\alpha \mathbf{k}_{\alpha}}-E_{\beta \mathbf{k}_{\beta}}+\omega+i \eta} \\
& \times \Psi_{\alpha \mathbf{k}_{\alpha}}\left(\mathbf{r}_{1}\right) \Psi_{\beta \mathbf{k}_{\beta}}^{*}\left(\mathbf{r}_{1}\right) \Psi_{\beta \mathbf{k}_{\beta}}\left(\mathbf{r}_{2}\right) \Psi_{\alpha \mathbf{k}_{\alpha}}^{*}\left(\mathbf{r}_{2}\right)
\end{aligned}
$$

In this equation $\alpha$ and $\beta$ indicate the band numbers, $\mathbf{k}_{\alpha}$ and $\mathbf{k}_{\beta}$ are wave vectors, and $\eta$ is an infinitesimally small positive constant.

The formalism outlined above does not contain temperature explicitly. However, the contributions to the decay rate $\Gamma_{e-e}$ are determined by electronic energies $E_{n, \mathbf{k}}$ and wave functions $\Psi_{n, \mathbf{k}}$. Thus, in order to study the thermal change of $\Gamma_{e-e}$, we take into account the temperature dependence of the electronic structure using a procedure, described below in Sec. II C.

\section{B. Electron-phonon scattering}

The contribution of the electron-phonon ( $e$-ph) interaction to the decay rate $\Gamma_{e \text {-ph }}$ is expressed in terms of the Eliashberg function $^{40} \alpha^{2} F(\omega)$ which accounts for phonon emission $(E)$ and absorption $(A)$ processes in scattering of electronic excitations:

$$
\begin{aligned}
\Gamma_{e-\mathrm{ph}}= & 2 \pi \int_{0}^{\omega_{\max }}\left\{\alpha^{2} F_{i, \mathbf{k}_{i}}^{A}(\omega)\left[n(\omega)+f\left(E_{i, \mathbf{k}_{i}}+\omega\right)\right]\right. \\
& \left.+\alpha^{2} F_{i, \mathbf{k}_{i}}^{E}(\omega)\left[1+n(\omega)-f\left(E_{i, \mathbf{k}_{i}}-\omega\right)\right]\right\} d \omega,
\end{aligned}
$$

where $n(\omega)$ and $f(\epsilon)$ are the Bose and Fermi functions, respectively, and $\omega_{\max }$ is the maximum phonon frequency. In the high-temperature limit the $e$-ph decay rate grows linearly with temperature $(T)$,

$$
\Gamma_{e-\mathrm{ph}}=2 \pi \lambda_{i, \mathbf{k}_{i}} T,
$$

where $\lambda_{i, \mathbf{k}_{i}}$ is the $e$-ph coupling parameter defined as

$$
\lambda_{i, \mathbf{k}_{i}}=\int_{0}^{\omega_{\max }} \frac{\alpha^{2} F_{i, \mathbf{k}_{i}}^{E}(\omega)+\alpha^{2} F_{i, \mathbf{k}_{i}}^{A}(\omega)}{\omega} d \omega .
$$

The Eliashberg spectral function of an electron or hole is defined as

$$
\begin{aligned}
\alpha^{2} F_{i, \mathbf{k}_{i}}^{A(E)}(\omega)= & \frac{1}{(2 \pi)^{2}} \int d^{2} \mathbf{q} \sum_{\nu, f} \delta\left(\omega-\omega_{\mathbf{q}, v}\right)\left|g_{i, f}\left(\mathbf{k}_{i}, \mathbf{k}_{f}, \mathbf{q}, \nu\right)\right|^{2} \\
& \times \delta\left(E_{i, \mathbf{k}_{i}}-E_{f, \mathbf{k}_{f}} \pm \omega_{\mathbf{q}, v}\right),
\end{aligned}
$$

where $g_{i, f}\left(\mathbf{k}_{i}, \mathbf{k}_{f}, \mathbf{q}, v\right)$ is the $e$-ph matrix element reproducing the probability of a transition from an initial state $\left(i, \mathbf{k}_{i}\right)$ to a final state $\left(f, \mathbf{k}_{f}\right)$ by emission or absorption of a phonon with a frequency $\omega_{\mathbf{q}, v}$ and momentum $\mathbf{q}$, which satisfies the relation

$$
\pm\left(\mathbf{k}_{f}-\mathbf{k}_{i}\right)=\mathbf{q}+\mathbf{G}_{\|} .
$$

Here "+" and "-" signs correspond to phonon absorption and emission processes, respectively. $\mathbf{G}_{\|}$is a two-dimensional reciprocal lattice vector. The summation in (9) is carried out over all possible final states $\Psi_{\mathbf{k}_{f}}$ and all phonon modes. The matrix element is defined by

$$
g_{i, f}\left(\mathbf{k}_{i}, \mathbf{k}_{f}, \mathbf{q}, \nu\right)=\left(\frac{1}{2 M \omega_{\mathbf{q}, v}}\right)^{1 / 2}\left\langle\Psi_{i, \mathbf{k}_{i}}\left|\hat{\varepsilon}_{\mathbf{q}, v} \cdot \nabla_{\mathbf{R}} V_{e-i}\right| \Psi_{f, \mathbf{k}_{f}}\right\rangle,
$$

with $M$ being the mass of an atom, $\hat{\varepsilon}_{\mathbf{q}, v}$ the phonon polarization vector, and $\nabla_{\mathbf{R}} V_{e-i}$ the gradient of an electron-ionic pseudopotential with respect to atomic positions $\mathbf{R}$.

To calculate the matrix element (11) we use the method developed in Refs. 22 and 37. This method consists of three 

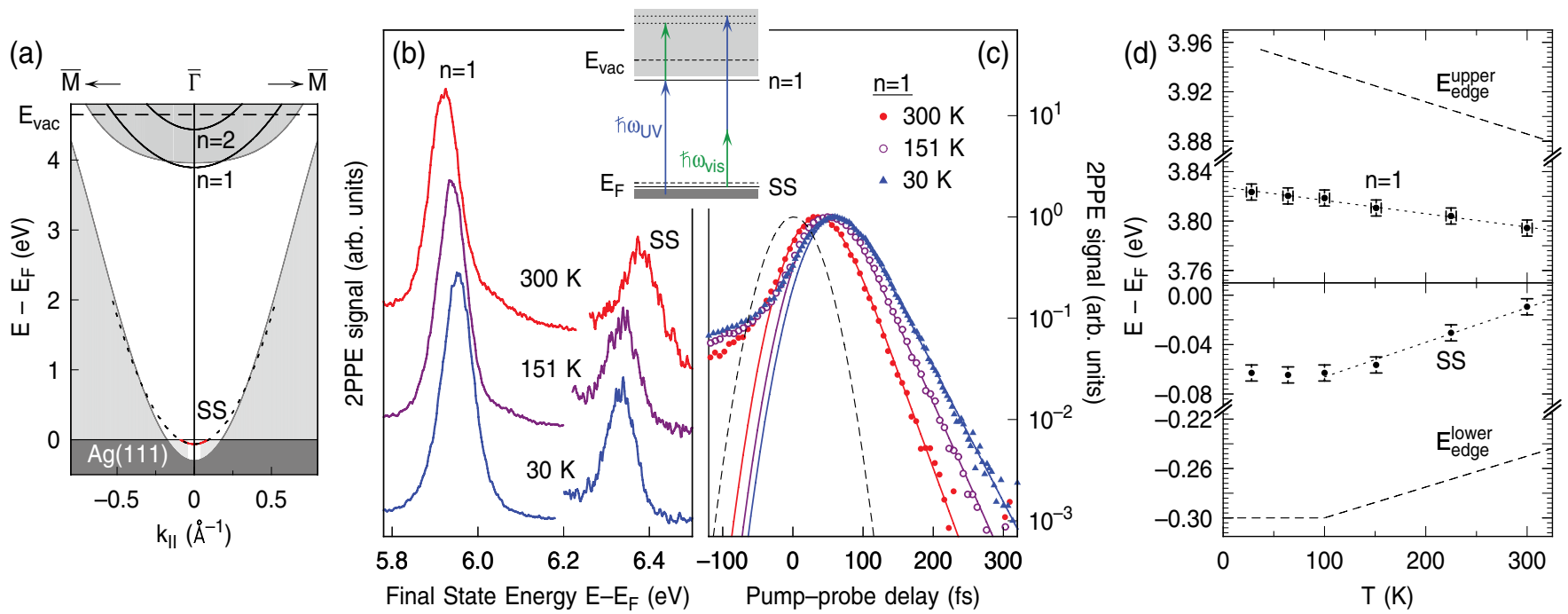

FIG. 1. (Color online) (a) Electronic surface band structure of $\mathrm{Ag}(111)$. Gray areas indicate projected bulk states. The dashed and solid lines represent the dispersion of the Shockley surface state (SS), the $(n=1)$ image-potential state, and the $(n=2)$ image-potential resonances, respectively. $E_{\mathrm{F}}$ and $E_{\mathrm{vac}}$ are Fermi and vacuum level, respectively. (b) Normalized 2PPE spectra as functions of the final-state energy with respect to the Fermi energy $E_{\mathrm{F}}$. The spectra were recorded at the indicated sample temperatures and analyzed for normal emission. The inset depicts the excitation schemes for the $(n=1)$ image-potential state and the Shockley surface state (SS). (c) Time-resolved pump-probe traces at the energies of the $(n=1)$ image-potential state for different sample temperatures. The solid lines are fits of a rate equation model. The dashed line represents the cross correlation of the laser pulses. (d) Temperature-dependent energy of the Shockley surface state (SS), the $(n=1)$ image-potential state, as well as of the lower $E_{\text {edge }}^{\text {lower }}$ and the upper $E_{\text {edge }}^{\text {upper }}$ edges of the projected bulk gap.

independent approximations: (i) the electron-ionic potential $V_{e-i}$ is taken to be the Ashkroft empty-core pseudopotential, screened in Thomas-Fermi approximation, (ii) phonon spectra are calculated on the basis of the embedded atom method derived interatomic potentials, ${ }^{41}$ and (iii) the electronic structure of the surface is calculated within a one-dimensional potential, ${ }^{42}$ described in Sec. II C. It should be noted, that the electronic energies and wave functions depend on temperature, and hence the matrix element (11), the Eliashberg function (9), and the $e$-ph coupling constant (8) are also functions of temperature.

\section{Model for the description of electronic states}

In order to describe the electronic energies $E_{n, \mathbf{k}}$ and wave functions $\Psi_{n, \mathbf{k}}$ we utilize a model pseudopotential, ${ }^{42,43}$ which varies only in the direction $z$ perpendicular to the surface and remains constant in the plane of the surface. The parameters of the pseudopotential are adjusted to reproduce the main features of the surface band structure at the $\bar{\Gamma}$ point: the energies of the lower $\left(E_{\text {edge }}^{\text {lower }}\right)$ and the upper $\left(E_{\text {edge }}^{\text {upper }}\right)$ edges of the projected bulk band gap, the Shockley surface state $E_{\mathrm{SS}}$, and the $(n=1)$ image-potential state $E_{n=1}$. This method has been widely used for calculations of the lifetimes of excitations in surface states and image-potential states on close-packed surfaces of various metals. ${ }^{44}$

The energies of the Shockley surface state and the $(n=1)$ image-potential state are measured in the present experiment at different temperatures in the range from 30 to $300 \mathrm{~K}$. To calculate the temperature-dependent edges of the energy gap we utilize the multiple-reflection model. ${ }^{9,45}$ This model allows one to calculate the positions of the Shockley state and the $(n=1)$ image state from the positions of the edges of the energy gap. ${ }^{9,45}$ In this work we solve the converse problem (analogous to Ref. 20) to calculate the energies of the gap edges from the energies of the Shockley state and the first image-potential state. Some estimations of the accuracy of such an approach should be made. The model was used in Ref. 46 to reproduce the experimentally measured temperature dependence of the energy $E_{\mathrm{SS}}(T)$ of the Shockley states on the (111) surfaces of noble metals. It was found, that this model accurately reproduces the slope $d E_{\mathrm{SS}} / d T$, while the calculated dependence $E_{\mathrm{SS}}(T)$ is shifted from the experimental data by $\sim 50 \mathrm{meV}$. Thus we may suppose that the multiple-reflection model reproduces the positions of the gap edges correctly with an accuracy of $\sim 50 \mathrm{meV}$.

The resulting values $E_{\text {edge }}^{\text {lower }}, E_{\text {edge }}^{\text {upper }}, E_{\mathrm{SS}}$, and $E_{n=1}$ are presented in Fig. 1(d). In order to account for the temperature dependence of the band structure, we adjust different sets of parameters of the pseudopotential for different temperatures in the range from 0 to $300 \mathrm{~K}$. Thus, the values $E_{\text {edge }}^{\text {lower }} E_{\text {edge }}^{\text {upper }}$, $E_{\mathrm{SS}}$, and $E_{n=1}$ calculated with the temperature-dependent pseudopotential coincide with those presented in Fig. 1(d).

\section{EXPERIMENT}

The experiments were performed in an ultrahigh vacuum chamber with a base pressure of $5 \times 10^{-11}$ mbar. $^{47,48} \mathrm{~A}$ Ti:sapphire laser amplifier system was used to pump an optical parametric amplifier which provided laser pulses with a photon energy of $\hbar \omega_{\text {vis }}=2.13 \mathrm{eV}$ and a pulse duration of around 55 fs. The output was split into two parts. One part was frequency doubled in order to obtain $\hbar \omega_{\mathrm{UV}}=4.27 \mathrm{eV} / 55 \mathrm{fs}$ laser pulses, while the second part was guided over a motordriven delay stage. Then the $p$-polarized laser pulses were 
aligned collinearly and focused onto the sample at an angle of incidence of $72^{\circ}$ relative to the surface normal. The photoemitted electrons were analyzed with respect to their kinetic energy and emission angle using a hemispherical electron analyzer with an angle-resolved lens mode (Specs Phoibos 150) and detected with a two-dimensional chargecoupled-device detector. ${ }^{49}$ The overall energy resolution of the $2 \mathrm{PPE}$ experiment was $\Delta E \lesssim 30 \mathrm{meV}$. The $\mathrm{Ag}(111)$ crystal was prepared by repeated sputtering and annealing cycles as described in Ref. 14. The surface quality was checked by $\mathrm{x}$-ray photoemission spectroscopy and low-energy electron diffraction.

\section{RESULTS AND DISCUSSION}

The schematic plot of the $\operatorname{Ag}(111)$ band structure is presented in Fig. 1(a). The Shockley surface state (SS) and the $(n=1)$ image-potential state are located in the projected bulk gap near the $\bar{\Gamma}$ point. Image-potential states with quantum numbers $n \geqslant 2$ are degenerate with projected bulk states, and hence they form image-potential resonances.

Figure 1(b) shows three 2PPE spectra at $\bar{\Gamma}$ in the energy region of the $(n=1)$ state and the Shockley state signals for sample temperatures of 300,151 , and $30 \mathrm{~K}$. The 2PPE spectra were normalized and scaled differently in the respective regions for better comparison. With increasing sample temperature, the signal of the $(n=1)$ state shifts to lower final-state energies. Simultaneously, a shift to higher energies is observed for the 2PPE signal of the Shockley state. The inset in Fig. 1 depicts the applied excitation scheme for the $(n=1)$ state and the Shockley state. Electrons are excited into the $(n=1)$ state by the UV pulses ( $\left.\hbar \omega_{\mathrm{UV}}\right)$ and subsequently photoemitted by absorption of photons $\hbar \omega_{\text {vis }}$. Electrons from the Shockley state are photoemitted in a direct, nonresonant 2PPE process with absorption of one photon of each pulse $\left(\hbar \omega_{\mathrm{UV}}+\hbar \omega_{\mathrm{vis}}\right)$. Taking into account the different photoemission pathways, the binding energies of the $(n=1)$ state and the Shockley state with respect to $E_{\mathrm{F}}$ are shown as functions of sample temperatures in Fig. 1(d). The temperature dependence of the energy of the Shockley surface state $E_{\mathrm{SS}}$ has a plateau at $T<100 \mathrm{~K}$ which is in good agreement with low-temperature measurements by means of scanning tunneling spectroscopy $[-65 \mathrm{meV}$ at $T=5 \mathrm{~K}$ (Ref. 50) and at $T=50 \mathrm{~K}$ (Ref. 51)]. The energy calibration of the 2PPE spectra was matched in such a way that the Shockley state is found at $E-E_{\mathrm{F}}=-0.065 \mathrm{meV}$ for low temperatures in accordance with results from photoemission spectroscopy [ $-60 \mathrm{meV}$ at $T=5 \mathrm{~K}$ (Ref. 46)]. For $T>100 \mathrm{~K}$ $E_{\mathrm{SS}}$ grows linearly with temperature. This dependence is also in agreement with data reported in Ref. 46. The energy of the $n=1$ image state $E_{n=1}$ decreases linearly from $3.83 \mathrm{eV}$ at $T=0 \mathrm{~K}$ to $3.80 \mathrm{eV}$ at $T=300 \mathrm{~K}$. This is in agreement with the previously reported value $E_{n=1}=E_{\mathrm{vac}}-0.77 \mathrm{eV}=$ $3.79 \mathrm{eV}$ (Ref. 52) for room temperature. [The work function $E_{\text {vac }}-E_{F}=4.56 \mathrm{eV}$ (Ref. 52) is used.]

The temperature-dependent dynamics of electrons excited into the $(n=1)$ state were investigated by time-resolved 2PPE. In the pump-probe traces that are shown in Fig. $1(\mathrm{c})$ the $(n=1)$ state intensity is plotted as function of the relative delay between the laser pulses. For negative pump-probe delays the photoemission pulses $\left(\hbar \omega_{\text {vis }}\right)$ arrive at the sample before

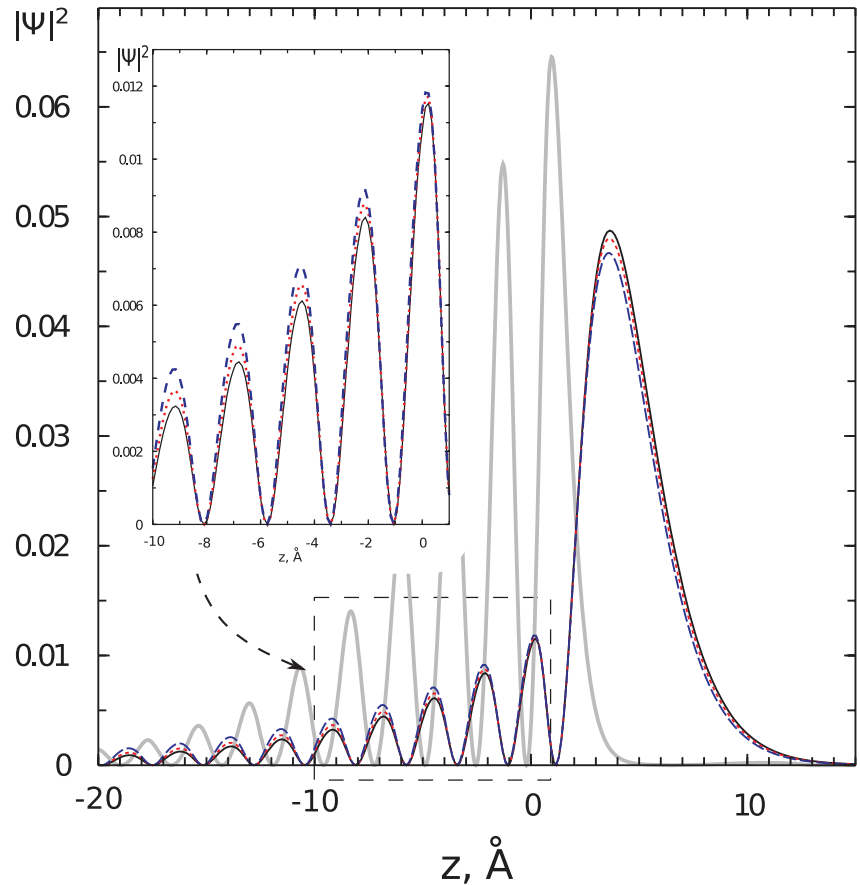

FIG. 2. (Color online) Probability distribution of the first imagepotential state at $T=28 \mathrm{~K}$ (solid line), $T=151 \mathrm{~K}$ (dotted line), and $T=300 \mathrm{~K}$ (dashed line), and the surface state at $T=300 \mathrm{~K}$ (light gray line), integrated over directions parallel to the surface. The $z$ axis is perpendicular to the surface; $z=0$ corresponds to the outermost atomic layer.

the UV excitation, while positive delay times correspond to delayed photoemission pulses. The lifetimes $\tau$ of the exponential decay of the excited $(n=1)$ state population has been extracted from the pump-probe traces by a fit using a rate equation model. This model includes the cross correlation of the laser pulses and an exponential population decay. The results of the fits are shown as thin solid lines in Fig. 1(c). The experimentally determined population decay rate is obtained from $\Gamma=\hbar / \tau$ and corresponds to the inelastic decay rate of the excited electrons. The experimental data show that not only the binding energy of the $(n=1)$ state is modified with increasing temperature, but also the decay rate increases. The signal which is observed for negative pump-probe delays originates from hot electrons. The nonthermal distribution is excited by the intense $\hbar \omega_{\text {vis }}$ pulse and electrons are photoemitted by absorbing UV photons. ${ }^{53}$

Using the multiple-reflection model, we calculate the temperature dependence of the positions of the edges of the projected bulk gap at the $\bar{\Gamma}$ point. ${ }^{9,20,45}$ The zerotemperature limits $E_{\text {edge }}^{\text {upper }}(T \rightarrow 0)=3.96 \mathrm{eV}$ and $E_{\text {edge }}^{\text {lower }}(T \rightarrow$ $0)=-0.30 \mathrm{eV}$ are not far from the result of first-principles calculations $E_{\text {edge }}^{\text {upper }}=3.9 \mathrm{eV}$ and $E_{\text {edge }}^{\text {lower }}=-0.40 \mathrm{eV}^{42}$

When the temperature is raised, the upper edge of the projected gap and the $(n=1)$ state move downward with the rates $d E_{\text {edge }}^{\text {upper }} / d T=-0.26 \mathrm{meV} / \mathrm{K}$ and $d E_{n=1} / d T=$ $-0.10 \mathrm{meV} / \mathrm{K}$, respectively. Thus, the $(n=1)$ state approaches the gap edge, and therefore its wave function is modified. As demonstrated in Fig. 2, at higher values of $T$ the main probability peak of the $(n=1)$ state is slightly decreased, while the weight inside the crystal is increased. 

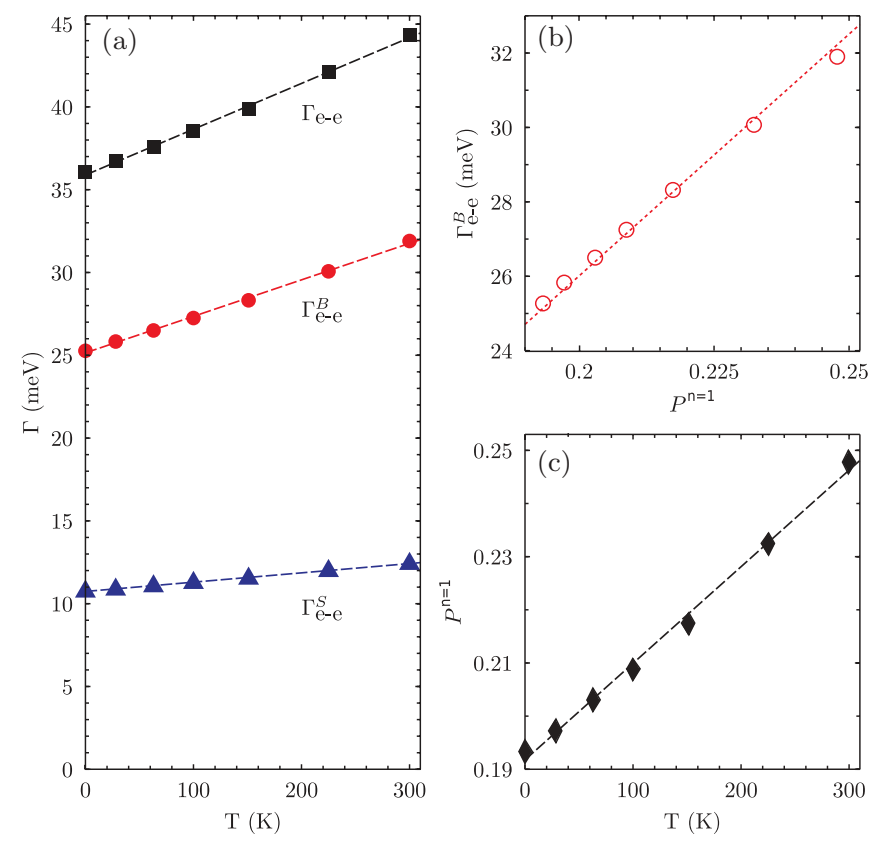

FIG. 3. (Color online) (a) Electron-electron scattering contribution $\Gamma_{e-e}$ to the decay rate of the $(n=1)$ image-potential state (filled squares) and contributions from scattering to bulk ( $\Gamma_{e-e}^{B}$, filled circles) and surface ( $\Gamma_{e-e}^{S}$, filled triangles) states; (b) dependence of the bulk contribution $\Gamma_{e-e}^{B}$ from the penetration integral $P^{n=1}$ (empty circles); and (c) penetration integral $P^{n=1}$ (filled diamonds) as functions of temperature. Dashed lines - linear approximation of the calculated data; dotted line in $b$ - proportional approximation $\Gamma_{e-e}^{B}=P^{n=1} \times 130 \mathrm{meV}$.

The inelastic electron-electron contribution to the decay rate of the $(n=1)$ image-potential state contains contributions from scattering into bulk electronic states $\Gamma_{e-e}^{B}$ and into the surface state $\Gamma_{e-e}^{S}$ [Fig. 3(a)]. $\Gamma_{e-e}^{S}$ contributes $30 \%$ to the total decay rate at $T=0$ and slightly grows with temperature: $d \Gamma_{e-e}^{S} / d T=0.006 \mathrm{meV} / \mathrm{K} . \Gamma_{e-e}^{B}$ increases considerably faster with temperature $\left(d \Gamma_{e-e}^{B} / d T=0.022 \mathrm{meV} / \mathrm{K}\right)$ and mostly determines the linear growth of the total decay rate $\Gamma_{e-e}$.

This is the direct consequence of the change of the wave function of the $(n=1)$ state. As follows from Eq. (2), $\Gamma_{e-e}$ is roughly proportional to the overlap between the charge densities of the initial state $\left|\Psi_{n=1}\right|^{2}$ and of the final states $\left|\Psi_{f, \mathbf{k}_{f}}\right|^{2}$. In the case of transitions into bulk states this overlap may be estimated by the penetration integral

$$
P^{n=1}=\int_{-\infty}^{a_{s} / 2}\left|\Psi_{n=1}(z)\right|^{2} d z,
$$

$a_{s}=2.35 \AA$ being the interlayer spacing of the silver in the [111] direction. Figure 3(b) demonstrates the proportionality between $P^{n=1}$ and $\Gamma_{e-e}^{B}$. As shown in Fig. 3(c), $P^{n=1}$ grows linearly with temperature, resulting in the linear growth of $\Gamma_{e-e}^{B}$.

As can be seen in Fig. 2, the $(n=1)$ wave function in the vicinity of $z=0$ (where the surface state is mostly localized) practically does not depend on temperature, and hence $\Gamma_{e-e}^{S}$ is weakly modified with temperature. This behavior is similar to that observed in the study of the wave-vector dependence of the decay rates of image states on $\mathrm{Cu}(111)$ and $\mathrm{Ag}(111):{ }^{48,54}$ The approach of the $(n=1)$ state to the gap edge results in deeper penetration of the $(n=1)$ wave function into the bulk and enhances the scattering into bulk states, while the scattering rate into the Shockley state remains practically constant.

We find that the electron-phonon scattering of electrons in the $(n=1)$ image-potential state is rather weak. The $e$-ph coupling constant (8) varies from $\lambda=4 \times 10^{-3}$ at $T=0$ to $\lambda=5 \times 10^{-3}$ at $T=300 \mathrm{~K}$ for $\operatorname{Ag}(111)$. These values are the same order of magnitude as for the $(n=1)$ image state on $\operatorname{Pd}(111)^{36}\left(\lambda=2 \times 10^{-3}\right), \mathrm{Cu}(100)^{34}\left(\lambda=10^{-2}\right), \operatorname{Ag}(100)^{34}$ $\left(\lambda=5 \times 10^{-3}\right)$, and the first two image states on $\operatorname{Pd}(110)^{35}$ $\left(\lambda=2 \times 10^{-3}\right)$. The resulting contribution to the decay rate $\Gamma_{e \text {-ph }}$ does not exceed $1 \mathrm{meV}$ up to room temperature, and one may expect that the contribution of the $e$-ph scattering to the dephasing rate is also very small.

However, a significantly larger value of the electron-phonon coupling constant $\lambda=6 \times 10^{-2}$, was reported for the $(n=1)$ image-potential state of $\mathrm{Cu}(111)$ by Knoesel et al ${ }^{20}$ Given the similar band structure of $\mathrm{Cu}(111)$ and $\mathrm{Ag}(111)$ surfaces this large difference between $e$-ph coupling strengths of $\mathrm{Cu}(111)$ and $\operatorname{Ag}(111)$ is surprising and deserves further attention. Therefore, we also calculated the $e$-ph coupling constant for the $(n=1)$ image state on $\mathrm{Cu}(111)$. We obtained the value $\lambda=7 \times 10^{-3}$, which is the same order of magnitude as for $\operatorname{Ag}(111)$, as well as for the other surfaces listed above. There are several reasons why the experiment of Ref. 20 might have overestimated the $e$-ph coupling constant $\lambda$. First of all, Knoesel et al. extracted $\lambda$ from the temperature dependence of the pure dephasing rate $T_{2}^{*}$ which was determined from a comparison of $2 \mathrm{PPE}$ decay rates and linewidths. ${ }^{20}$ For small $T_{2}^{*}$ times, however, this method is generally less straightforward than, e.g., quantum-beat spectroscopy or direct methods of determining quasielastic electron-scattering rates. ${ }^{55-57}$ Moreover, the increase of the pure dephasing rate with temperature can have other origins than coupling to phonons. Many defects are known to scatter image-potential electrons efficiently. ${ }^{58}$ Stronger penetration of the image state wave function into the bulk at higher temperature, discussed above, is expected to lead to an increase of the defect scattering rate with temperature. Also thermally activated defects ${ }^{19,24}$ contribute to the temperature dependence of the scattering rate.

The temperature dependence of the calculated $\left(\Gamma_{\mathrm{Th}}=\right.$ $\left.\Gamma_{e-e}+\Gamma_{e-\mathrm{ph}}\right)$ and measured $\left(\Gamma_{\mathrm{Ex}}\right)$ decay rates of the imagepotential state is shown in Fig. 4. Both, theory and experiment show linear growth of the decay rate with temperature. The calculated slope $d \Gamma_{\mathrm{Th}} / d T=0.030 \mathrm{meV} / \mathrm{K}$ is also in agreement with the value $d \Gamma_{\mathrm{Ex}} / d T=0.034 \mathrm{meV} / \mathrm{K}$, obtained by linear fitting the experimental data. However, $\Gamma_{\mathrm{Th}}$ is approximately $16 \mathrm{meV}$ larger than $\Gamma_{\mathrm{Ex}}$ over the whole temperature range considered. There may be two reasons for such discrepancy.

First, $\Gamma_{\mathrm{Th}}$ may be overestimated because of neglecting the $d$ electrons in the present calculations. The inclusion of $d$ electrons decreases the surface plasmon energy below the energy of $(n=1)$ state on $\operatorname{Ag}(111) .{ }^{59,60}$ Although this opens an extra inelastic decay channel for electrons in the image-potential state, it has been shown ${ }^{59,60}$ that the lifetimes of image states are increased due to the strongly nonlocal character of the self-energy near the surface. For example, the surface plasmon channel reduces the decay rate of the $(n=1)$ image-potential state on $\mathrm{Ag}(111)$ by $8 \mathrm{meV}^{60}$ 


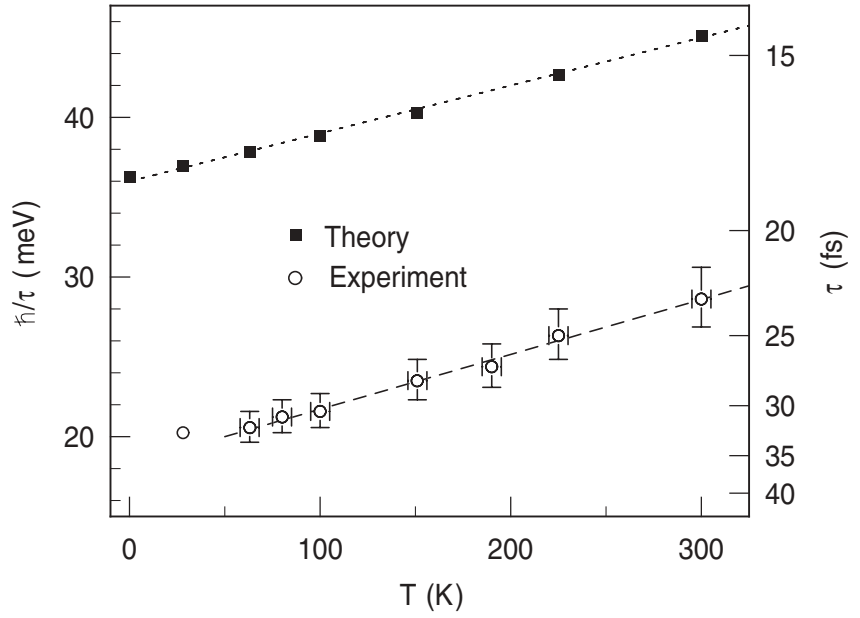

FIG. 4. The measured (open dots) and calculated (filled squares) decay rate of the $n=1$ image-potential state on $\operatorname{Ag}(111)$ as a function of temperature. Dashed (dotted) lines represent linear interpolations of the experimental (theoretical) data. The slopes are $0.034 \mathrm{meV} / \mathrm{K}$ $(0.030 \mathrm{meV} / \mathrm{K})$.

Second, the overestimation of $\Gamma_{\mathrm{Th}}$ may be attributed to the uncertainty of the determination of the upper gap edge position. According to our calculations, the main reason for the thermal shortening of the lifetimes is the change of the penetration of the $(n=1)$ wave function into the bulk metal due to the approach of the image state to the upper gap edge. The decay rate depends linearly on the energy difference $E_{\text {edge }}^{\text {upper }}-E_{n=1}$, while the derivative $d \Gamma_{\mathrm{Th}} / d\left(E_{\text {edge }}^{\text {upper }}-E_{n=1}\right)=-0.18$. Thus, a $50 \mathrm{meV}$ inaccuracy in the determination of $E_{\text {edge }}^{\text {upper }}-E_{n=1}$ may cause an inaccuracy $\sim 10 \mathrm{meV}$ in the calculated decay rate of the $(n=1)$ image state. However, such an inaccuracy only shifts the $\Gamma_{\mathrm{Th}}(T)$ dependence by a certain value, while leaving the slope $d \Gamma_{\mathrm{Th}} / d T$ unchanged.

\section{SUMMARY}

In summary, we have investigated the temperature dependence of the lifetime of electrons in the $n=1$ image potential state on the $\operatorname{Ag}(111)$ surface by means of timeresolved two-photon photoemission spectroscopy and manybody calculations. 2PPE experiments show that the decay rate $\Gamma$ of the $(n=1)$ image-potential state grows linearly with temperature. Theoretical investigations confirm this result and reveal the origin of such behavior: the shortening of the lifetime is caused by the increase of the electron-electron scattering rate, due to deeper penetration of the image state wave function into the bulk metal at higher temperature. The contribution of electron-phonon scattering to the decay rate was found to be very small. The calculated derivative $d \Gamma_{\mathrm{Th}} / d T=0.030 \mathrm{meV} / \mathrm{K}$ is close to the experimental value $d \Gamma_{\mathrm{Ex}} / d T=0.034 \mathrm{meV} / \mathrm{K}$.

\section{ACKNOWLEDGMENTS}

We thank P. M. Echenique for valuable discussions and acknowledge funding by the Deutsche Forschungsgemeinschaft through Grants No. GRK 790 and No. GU 495/2, the Ikerbasque Foundation, the University of the Basque Country (Project No. GV-UPV/EHU, Grant No. IT-366-07), and Ministerio de Ciencia e Inovación (Grant No. FIS201019609-C02-00).
${ }^{1}$ Laser Spectroscopy and Photochemistry on Metal Surfaces, edited by H. L. Dai and W. Ho (World Scientific, Singapore, 1995).

${ }^{2}$ H. Nienhaus, Surf. Sci. Rep. 45, 1 (2002).

${ }^{3}$ M. Bonn, S. Funk, Ch. Hess, D. N. Denzler, C. Stampfl, M. Scheffler, M. Wolf, and G. Ertl, Science 285, 1042 (1999).

${ }^{4}$ E. V. Chulkov, V. M. Silkin, and M. Machado, Surf. Sci. 482-485, 693 (2001).

${ }^{5}$ L. Vitali, P. Wahl, M. A. Schneider, K. Kern, V. M. Silkin, E. V. Chulkov, and P. M. Echenique, Surf. Sci. 523, L47 (2003).

${ }^{6}$ U. Höfer, I. L. Shumay, Ch. Reuß, U. Thomann, W. Wallauer, and Th. Fauster, Science 277, 1480 (1997).

${ }^{7}$ S. Link, H. A. Dürr, G. Bihlmayer, S. Blügel, W. Eberhardt, E. V. Chulkov, V. M. Silkin, and P. M. Echenique, Phys. Rev. B 63, 115420 (2001).

${ }^{8}$ A. Schäfer, I. L. Shumay, M. Wiets, M. Weinelt, T. Fauster, E. V. Chulkov, V. M. Silkin, and P. M. Echenique, Phys. Rev. B 61, 13159 (2000).

${ }^{9}$ P. M. Echenique and J. B. Pendry, J. Phys. C: Solid State Phys. 11, 2065 (1978).

${ }^{10}$ Th. Fauster and W. Steinmann, in Electromagnetic Waves: Recent Development in Research, edited by P. Halevi (Elsevier, Amsterdam, 1995), p. 350.

${ }^{11}$ P. M. Echenique, R. Berndt, E. V. Chulkov, Th. Fauster, A. Goldmann, and U. Höfer, Surf. Sci. Rep. 52, 219 (2004).
${ }^{12}$ A. G. Borisov, E. V. Chulkov, and P. M. Echenique, Phys. Rev. B 73, 073402 (2006).

${ }^{13}$ M. Winter, E. V. Chulkov, and U. Höfer, Phys. Rev. Lett. 107, 236801 (2011).

${ }^{14}$ M. Marks, C. H. Schwalb, K. Schubert, J. Güdde, and U. Höfer, Phys. Rev. B 84, 245402 (2011).

${ }^{15}$ A. Eiguren, B. Hellsing, F. Reinert, G. Nicolay, E. V. Chulkov, V. M. Silkin, S. Hüfner, and P. M. Echenique, Phys. Rev. Lett. 88, 066805 (2002).

${ }^{16} \mathrm{P}$. Straube, F. Pforte, T. Michalke, K. Berge, A. Gerlach, and A. Goldmann, Phys. Rev. B 61, 14072 (2000).

${ }^{17}$ S. M. Dounce, M. Yang, and H.-L. Dai, Phys. Rev. B 67, 205410 (2003).

${ }^{18}$ B. A. McDougall, T. Balasubramanian, and E. Jensen, Phys. Rev. B 51, 13891 (1995).

${ }^{19}$ M. Fuglsang Jensen, T. K. Kim, S. Bengió, I. Yu. Sklyadneva, A. Leonardo, S. V. Eremeev, E. V. Chulkov, and Ph. Hofmann, Phys. Rev. B 75, 153404 (2007).

${ }^{20}$ E. Knoesel, A. Hotzel, and M. Wolf, J. Electron Spectrosc. Relat. Phenom. 88-91, 577 (1998).

${ }^{21}$ J. J. Paggel, T. Miller, and T.-C. Chiang, Phys. Rev. Lett. 83, 1415 (1999).

${ }^{22}$ A. Eiguren, B. Hellsing, E. V. Chulkov, and P. M. Echenique, Phys. Rev. B 67, 235423 (2003). 
${ }^{23}$ S.-J. Tang, J. Shi, B. Wu, P. T. Sprunger, W. L. Yang, V. Brouet, X. J. Zhou, Z. Hussain, Z.-X. Shen, Z. Zhang, and E. W. Plummer, Phys. Status Solidi B 241, 2345 (2004).

${ }^{24}$ S. V. Eremeev and E. V. Chulkov, Phys. Solid State 51, 854 (2009).

${ }^{25}$ S. V. Eremeev, I. Yu. Sklyadneva, P. M. Echenique, S. D. Borisova, G. Benedek, G. G. Rusina, and E. V. Chulkov, Surf. Sci. 601, 4553 (2007).

${ }^{26}$ C. Kirkegaard, T. K. Kim, and Ph. Hofmann, New J. Phys. 7, 99 (2005).

${ }^{27}$ S. V. Eremeev, S. S. Tsirkin, and E. V. Chulkov, J. Exp. Theor. Phys. 110, 788 (2010).

${ }^{28}$ I. Yu. Sklyadneva, R. Heid, V. M. Silkin, A. Melzer, K.-P. Bohnen, P. M. Echenique, Th. Fauster, and E. V. Chulkov, Phys. Rev. B 80, 045429 (2009).

${ }^{29}$ E. Rotenberg and S. D. Kevan, J. Electron Spectrosc. Relat. Phenom. 126, 125 (2002).

${ }^{30} \mathrm{Ph}$. Hofmann, I. Yu. Sklyadneva, E. D. L. Rienks, and E. V. Chulkov, New J. Phys. 11, 125005 (2009).

${ }^{31}$ M. Hengsberger, R. Frésard, D. Purdie, P. Segovia, and Y. Baer, Phys. Rev. B 60, 10796 (1999).

${ }^{32}$ S. V. Eremeev, S. S. Tsirkin, and E. V. Chulkov, Phys. Rev. B 82, 035424 (2010).

${ }^{33}$ J. Kröger, Rep. Prog. Phys. 69, 899 (2006).

${ }^{34}$ A. Eiguren, B. Hellsing, E. V. Chulkov, and P. M. Echenique, J. Electron Spectrosc. Relat. Phenom. 129, 111 (2003).

${ }^{35}$ S. S. Tsirkin and S. V. Eremeev, Russ. Phys. J. 54, 92 (2011).

${ }^{36}$ I. Yu. Sklyadneva, S. S. Tsirkin, S. V. Eremeev, R. Heid, K.-P. Bohnen, and E. V. Chulkov, Phys. Solid State 53, 2508 (2011).

${ }^{37}$ B. Hellsing, A. Eiguren, and E. V. Chulkov, J. Phys.: Condens. Matter 14, 5959 (2002).

${ }^{38}$ I. Yu. Sklyadneva, R. Heid, K.-P. Bohnen, and E. V. Chulkov, New J. Phys. 11, 103038 (2009).

${ }^{39}$ L. Hedin, Phys. Rev. 139, A796 (1965).

${ }^{40} \mathrm{G}$. Grimvall, The Electron-Phonon Interaction in Metals (NorthHolland, Amsterdam, 1981).

${ }^{41}$ M. S. Daw and M. I. Baskes, Phys. Rev. B 29, 6443 (1984).
${ }^{42}$ E. V. Chulkov, V. M. Silkin, and P. M. Echenique, Surf. Sci. 437, 330 (1999).

${ }^{43}$ E. V. Chulkov, V. M. Silkin, and P. M. Echenique, Surf. Sci. 391, 11217 (1997)

${ }^{44}$ E. V. Chulkov, A. G. Borisov, J. P. Gauyacq, D. Sánchez-Portal, V. M. Silkin, V. P. Zhukov, and P. M. Echenique, Chem. Rev. 106, 4160 (2006).

${ }^{45}$ N. V. Smith, Phys. Rev. B 32, 3549 (1985).

${ }^{46}$ R. Paniago, R. Matzdorf, G. Meister, and A. Goldmann, Surf. Sci. 336, 113 (1995).

${ }^{47}$ A. Damm, K. Schubert, J. Güdde, and U. Höfer, Phys. Rev. B 80, 205425 (2009).

${ }^{48}$ K. Schubert, A. Damm, S. V. Eremeev, M. Marks, M. Shibuta, W. Berthold, J. Güdde, A. G. Borisov, S. S. Tsirkin, E. V. Chulkov, and U. Höfer, Phys. Rev. B 85, 205431 (2012).

${ }^{49}$ M. Rohleder, K. Duncker, W. Berthold, J. Güdde, and U. Höfer, New J. Phys. 7, 103 (2005).

${ }^{50}$ O. Jeandupeux, L. Bürgi, A. Hirstein, H. Brune, and K. Kern, Phys. Rev. B 59, 15926 (1999).

${ }^{51}$ J. Li, W.-D. Schneider, and R. Berndt, Phys. Rev. B 56, 7656 (1997)

${ }^{52}$ K. Giesen, F. Hage, F. J. Himpsel, H. J. Riess, and W. Steinmann, Phys. Rev. B 33, 5241 (1986).

${ }^{53}$ S. Ogawa, H. Nagano, and H. Petek, Phys. Rev. B 55, 10869 (1997).

${ }^{54}$ S. V. Eremeev, S. S. Tsirkin, and E. V. Chulkov, Phys. Solid State 52, 1768 (2010).

${ }^{55}$ S. Ogawa, H. Nagano, H. Petek, and A. P. Heberle, Phys. Rev. Lett. 78, 1339 (1997).

${ }^{56}$ Ch. Reuß, I. L. Shumay, U. Thomann, M. Kutschera, M. Weinelt, Th. Fauster, and U. Höfer, Phys. Rev. Lett. 82, 153 (1999).

${ }^{57}$ J. Güdde, M. Rohleder, T. Meier, S. W. Koch, and U. Höfer, Science 318, 1287 (2007).

${ }^{58}$ Th. Fauster, M. Weinelt, and U. Höfer, Planet. Space Sci. 82, 224 (2007).

${ }^{59}$ A. García-Lekue, J. M. Pitarke, E. V. Chulkov, A. Liebsch, and P. M. Echenique, Phys. Rev. Lett. 89, 096401 (2002).

${ }^{60}$ A. García-Lekue, J. M. Pitarke, E. V. Chulkov, A. Liebsch, and P. M. Echenique, Phys. Rev. B 68, 045103 (2003). 